\title{
A REGULARITY RESULT FOR A CONVEX FUNCTIONAL AND BOUNDS FOR THE SINGULAR SET
}

\author{
Bruno De Maria ${ }^{1}$
}

\begin{abstract}
In this paper we prove a regularity result for local minimizers of functionals of the Calculus of Variations of the type

$$
\int_{\Omega} f(x, D u) \mathrm{d} x
$$

where $\Omega$ is a bounded open set in $\mathbb{R}^{n}, u \in W_{\text {loc }}^{1, p}\left(\Omega ; \mathbb{R}^{N}\right), p>1, n \geq 2$ and $N \geq 1$. We use the technique of difference quotient without the usual assumption on the growth of the second derivatives of the function $f$. We apply this result to give a bound on the Hausdorff dimension of the singular set of minimizers.
\end{abstract}

Mathematics Subject Classification. 35J50, 35J60, 35B65.

Received February 4, 2009. Revised May 6, 2009.

Published online August 11, 2009.

\section{INTRODUCTION AND MAIN RESULTS}

In this paper we prove a regularity result for local minimizers of integral functionals of the Calculus of Variations of the type:

$$
\mathcal{F}(u ; \Omega):=\int_{\Omega} f(x, D u) \mathrm{d} x
$$

defined for Sobolev maps $u \in W^{1, p}\left(\Omega ; \mathbb{R}^{N}\right), p>1$. Here for $n \geq 2$ and $N \geq 1, \Omega$ is a bounded open set in $\mathbb{R}^{n}$ and $f: \Omega \times \mathbb{R}^{n N} \rightarrow \mathbb{R}$ is a continuous function. The assumptions we are going to consider are the following.

There exist positive constants $L, \nu, c>0$ such that for every $p>1$

$$
\begin{aligned}
\left(1+|\xi|^{2}\right)^{\frac{p}{2}} \leq f(x, \xi) & \leq L\left(1+|\xi|^{2}\right)^{\frac{p}{2}} ; \\
f\left(x, \frac{\xi_{1}+\xi_{2}}{2}\right) \leq & \frac{1}{2} f\left(x, \xi_{1}\right)+\frac{1}{2} f\left(x, \xi_{2}\right)-\nu\left(1+\left|\xi_{1}\right|^{2}+\left|\xi_{2}\right|^{2}\right)^{\frac{p-2}{2}}\left|\xi_{1}-\xi_{2}\right|^{2} ; \\
& \left|f_{\xi}\left(x_{1}, \xi\right)-f_{\xi}\left(x_{2}, \xi\right)\right| \leq c\left|x_{1}-x_{2}\right|^{\alpha}\left(1+|\xi|^{2}\right)^{\frac{p-1}{2}} ;
\end{aligned}
$$

for any $\xi, \xi_{1}, \xi_{2} \in \mathbb{R}^{n N}, x, x_{1}, x_{2} \in \Omega$ and $\alpha \in(0,1)$.

Keywords and phrases. Partial regularity, singular sets, fractional differentiability, variational integrals.

${ }^{1}$ Dipartimento di Matematica e Applicazioni "R. Caccioppoli" Università di Napoli "Federico II" Via Cintia, 80126 Napoli, Italy. bruno.demaria@dma.unina.it 
Assumption ( $\mathrm{H} 2)$ is a uniform convexity condition for the function $f$ with respect to the variable $\xi$, while with assumption (H3) we are requiring the vector field $x \in \Omega \rightarrow f_{\xi}(x, \xi)$ to be Hölder continuous for some exponent $\alpha \in(0,1)$ and for every $\xi \in \mathbb{R}^{n N}$.

Since the functional (1.1) is convex there is no difference between minimizers and critical points, i.e. minimizers are precisely the weak solutions to the Euler system $\operatorname{div} f_{\xi}(x, D u)=0$. Moreover if we require $f(\cdot, \xi) \in C^{2}$, so that (see Lem. 2.1 in the next section) (H2) is equivalent to the following ellipticity condition

$$
\left\langle f_{\xi \xi}(x, \xi) \lambda, \lambda\right\rangle \geq \nu|\lambda|^{2}\left(1+|\xi|^{2}\right)^{\frac{p-2}{2}} \quad \forall x \in \Omega, \forall \xi, \lambda \in \mathbb{R}^{n N},
$$

the Euler system turns out to be elliptic. There is a vast literature on the study of the regularity for weak solutions to this kind of systems and on the analogous issue for minimizers of functionals of the type $\mathcal{F}$ (see, for example, $[1,2,12,13,16]$; see also [23] for a nice survey on the subject). The usual technique to get existence of second derivatives when the elliptic system is not differentiable consists in constructing a suitable test function using difference quotient and then in reading the Hölder condition on $f$ as a kind of fractional differentiability (see also $[10,19,20])$. In this way it is possible to show that the gradient of $u$ belongs to some suitable fractional order Sobolev space. This technique has been used, for example, in [21] where the weak solutions to elliptic systems like

$$
\operatorname{div} a(x, D u)=0
$$

with $a: \Omega \times \mathbb{M}^{N \times n} \rightarrow \mathbb{M}^{N \times n}$, have been studied under the following growth, ellipticity and continuity assumptions:

$$
\begin{aligned}
\left|D_{\xi} a(x, \xi)\right| & \leq L\left(1+|\xi|^{2}\right)^{\frac{p-2}{2}}, \\
L^{-1}|\lambda|^{2}\left(1+|\xi|^{2}\right)^{\frac{p-2}{2}} & \leq \frac{\partial a_{i}^{k}}{\partial \xi_{j}^{h}}(x, \xi) \lambda_{i}^{k} \lambda_{j}^{h}, \\
\left|a(x, \xi)-a\left(x_{0}, \xi\right)\right| & \leq L\left|x-x_{0}\right|^{\alpha}\left(1+|\xi|^{2}\right)^{\frac{p-1}{2}}
\end{aligned}
$$

for any $z, \lambda \in \mathbb{M}^{n \times N}$ and $x, x_{0} \in \Omega$, where $p \geq 2, L \in(1,+\infty)$ and $\alpha \in(0,1)$ (see also [22]). As usual, a key role in the proof of the existence of fractional derivatives is played by assumption (1.4a) that in the case $a(x, \xi)=f_{\xi}(x, \xi)$ becomes in turn an assumption on the growth of second derivatives of $f$,

$$
\left|D^{2} f(x, \xi)\right| \leq L\left(1+|\xi|^{2}\right)^{\frac{p-2}{2}}
$$

Actually the main purpose of this paper is to provide a regularity result without any assumption on the growth of $D^{2} f$. This result relies essentially on a fundamental approximation procedure first introduced in [14].

In the case $p \geq 2$, our main result is the following.

Theorem 1.1. Let $f$ satisfy the assumptions (H1), (H2) and (H3), with $p \geq 2$. If the function $u \in W^{1, p}\left(\Omega ; \mathbb{R}^{N}\right)$ is a local minimizer of $\mathcal{F}$ in $\Omega$ then for every $B_{\rho} \subset B_{R} \subset \subset \Omega$ we have that

$$
D u \in W^{\frac{k}{p-1}, p}\left(B_{\rho} ; \mathbb{R}^{n N}\right) \cap L^{\frac{n p}{n-k q}}\left(B_{\rho} ; \mathbb{R}^{n N}\right)
$$

for every $k \in(0, \alpha)$, where $q \equiv \frac{p}{p-1}$ and

$$
\|\left. D u\right|_{L^{\frac{n p}{n-k q}\left(B_{\rho}\right)}} \leq c\left(\int_{B_{R}}\left(1+|D u(x)|^{p}\right) \mathrm{d} x\right)^{\frac{1}{2}},
$$

with $c \equiv c(n, N, L, \nu, R, \rho, \alpha, k, p)$. 
As far as we know, also in [11] the authors use the difference quotient method without the assumption (1.5) (or (1.4a)) but our higher integrability exponent is greater than the one found in [11] where the anisotropic growth conditions $1<p \leq q<p\left(\frac{n+\alpha}{n}\right)$ were examined (see also [9] for the autonomous case and [5,25] for polyconvex functionals). Here, the improved regularity stated in Theorem 1.1 depends on the assumption $p \geq 2$. In fact, in the case $1<p<2$ our higher integrability exponent is slightly greater than the one obtained in [11] in case $\alpha \geq \frac{1}{2}$ while is again better if $\alpha<\frac{1}{2}$. More precisely we have:

Theorem 1.2. Let $f$ satisfy the assumptions $(H 1)$, $(H 2)$ and $(H 3)$, with $1<p<2$. If the function $u \in$ $W^{1, p}\left(\Omega ; \mathbb{R}^{N}\right)$ is a local minimizer of $\mathcal{F}$ in $\Omega$ then for every $B_{\rho} \subset B_{R} \subset \subset \Omega$ we have that

$$
\left(1+|D u(x)|^{2}\right)^{\frac{p-2}{4}} D u(x) \in W^{k, 2}\left(B_{\rho} ; \mathbb{R}^{n N}\right) \cap L^{\frac{2 n}{n-2 k}}\left(B_{\rho} ; \mathbb{R}^{n N}\right)
$$

for every $0<k<\min \left\{\alpha, \frac{1}{2}\right\}$. As a consequence

$$
D u \in W^{k, p}\left(B_{\rho} ; \mathbb{R}^{n N}\right) \cap L^{\frac{n p}{n-2 k}}\left(B_{\rho} ; \mathbb{R}^{n N}\right)
$$

for every $0<k<\min \left\{\alpha, \frac{1}{2}\right\}$ and

$$
\|D u\|_{L^{\frac{n p}{n-2 k}\left(B_{\rho}\right)}} \leq c\left(\int_{B_{R}}\left(1+|D u(x)|^{p}\right) \mathrm{d} x\right)^{\frac{1}{2}},
$$

with $c \equiv c(n, N, L, \nu, R, \rho, \alpha, k, p)$.

The proof of these two theorems is divided in two steps. In the first step we assume that $f(\cdot, \xi) \in C^{2}$ but we are able to establish the estimates (1.6) and (1.9) independently of the $C^{2}$ norm of the integrand $f$, by adopting an argument first used in [14]. In the second step we remove the assumption $f(\cdot, \xi) \in C^{2}$ using an approximation procedure introduced in [14] and developed in $[7,10,15]$. More precisely we approximate $f$ by a sequence $\left\{f_{h}\right\}$ of $C^{2}$ functions which are strictly elliptic (and the ellipticity constant is precisely the $\nu$ appearing in $(H 2))$. The minimizers $\left\{u_{h}\right\}$ of $\left\{f_{h}\right\}$ all satisfy estimates (1.6) and (1.9). More important the estimates are independent of the $C^{2}$ norm of $\left\{f_{h}\right\}$ and thus are preserved in passing to the limit. Hence a control of the type

$$
\left|D^{2} f(x, \xi)\right| \leq c\left(1+|\xi|^{2}\right)^{\frac{p-2}{2}}, \quad \forall(x, \xi) \in \Omega \times \mathbb{R}^{n},
$$

on the growth of second derivatives of $f$ never enters into play. It is worth pointing out that our crucial estimates (1.6) and (1.9) are consequences of some nice embedding properties enjoyed by fractional order Sobolev spaces (see [3]).

We remark that the cases $1<p<2$ and $p \geq 2$ have different technical difficulties and therefore they have to be treated separately. The subquadratic case has been treated in [4] for the first time, in the quasiconvex setting; however the paper [4] does not deal with the full case $1<p<2$ but only with $2 n /(n+2)<p<2$. The extension to the full interval $1<p<2$ has been achieved in the subsequent paper [6].

Moreover we would like to notice that in the case $p=2$ we recover the same regularity of [21] without the growth assumption on the second derivatives (1.4a).

In the final part of this paper we apply the previous results to get a bound on the Hausdorff dimension of the singular set.

\section{NOTATIONS AND PRELIMINARIES}

In this section we explain the notations used in the paper and recall some useful results needed for the proof of Theorems 1.1 and 1.2 that will be given in the next section. 
We shall indicate with $B_{R}\left(x_{0}\right)$ the ball centered at the point $x_{0} \in \mathbb{R}^{n}$ and having radius $R>0$. We shall omit the center of the ball when no confusion arises. All the balls considered will be concentric unless differently specified.

As usual $\left\{e_{s}\right\}_{1 \leq s \leq n}$ is the standard basis in $\mathbb{R}^{n}$ and if $u, v \in \mathbb{R}^{k}$ the tensor product $u \otimes v \in \mathbb{R}^{k^{2}}$ of $u$ and $v$ is defined by $(u \otimes v)_{i, j}:=v_{i} w_{j}$.

In the estimates $c$ is a constant, depending on the data of the problem, that may change from line to line.

Let us recall the following definition of local minimizer for the functional $\mathcal{F}$.

Definition 2.1. A map $u \in W^{1, p}\left(\Omega ; \mathbb{R}^{N}\right)$ is a local minimizer of the functional $\mathcal{F}$ if

$$
\mathcal{F}(u ; A) \leq \mathcal{F}(v ; A)
$$

whenever $A \subset \subset \Omega$ and $u-v \in W_{0}^{1, p}\left(A ; \mathbb{R}^{N}\right)$.

As we already said we need the machinery of fractional order Sobolev spaces. These spaces are defined as follows.

Definition 2.2. If $A$ is a smooth, bounded open subset of $\mathbb{R}^{n}$ and $\theta \in(0,1), 1 \leq p<+\infty$ a function $u$ belongs to the fractional order Sobolev space $W^{\theta, q}\left(A ; \mathbb{R}^{n}\right)$ if and only if

$$
\|u\|_{W^{\theta, p}}:=\left(\int_{A}|u(x)|^{p} \mathrm{~d} x\right)^{\frac{1}{p}}+\left(\int_{A} \int_{A} \frac{|u(x)-u(y)|^{p}}{|x-y|^{n+p \theta}} \mathrm{d} x \mathrm{~d} y\right)^{\frac{1}{p}} .
$$

This quantity is a norm making $W^{\theta, p}\left(A ; \mathbb{R}^{n}\right)$ a Banach space.

In the context of fractional order Sobolev spaces we have to use fractional difference quotient. Therefore we introduce the following finite difference operator.

Definition 2.3. For every vector valued function $F: \mathbb{R}^{n} \rightarrow \mathbb{R}^{N}$ the finite difference operator is defined by

$$
\tau_{s, h} F(x)=F\left(x+h e_{s}\right)-F(x)
$$

where $h \in \mathbb{R}, e_{s}$ is the unit vector in the $x_{s}$ direction and $s \in\{1, \ldots, n\}$.

The following proposition describes some elementary properties of the finite difference operator and can be found, for example, in [17].

Proposition 2.1. Let $f$ and $g$ be two functions such that $F, G \in W^{1, p}(\Omega)$, with $p \geq 1$, and let us consider the set

$$
\Omega_{|h|}:=\{x \in \Omega: \operatorname{dist}(x, \partial \Omega)>|h|\}
$$

Then

(d1) $\tau_{s, h} F \in W^{1, p}(\Omega)$ and

$$
D_{i}\left(\tau_{s, h} F\right)=\tau_{s, h}\left(D_{i} F\right) .
$$

(d2) If at least one of the functions $F$ or $G$ has support contained in $\Omega_{|h|}$ then

$$
\int_{\Omega} F \tau_{s, h} G \mathrm{~d} x=-\int_{\Omega} G \tau_{s,-h} F \mathrm{~d} x
$$

(d3) We have

$$
\tau_{s, h}(F G)(x)=F\left(x+h e_{s}\right) \tau_{s, h} G(x)+G(x) \tau_{s, h} F(x) .
$$


The following statement has been proved in [15]. It states that the condition of uniform convexity of the functional $\mathcal{F}$ is equivalent to the ellipticity condition for the Euler system of $\mathcal{F}$.

Lemma 2.1. Let $f: \mathbb{R}^{n N} \rightarrow[0,+\infty)$ be a $C^{2}$ function and $p>1$. Then $f$ satisfies $(H 2)$ if and only if there exists a constant $c_{0}$ such that for all $\xi \in \mathbb{R}^{n N}$

$$
\left\langle f_{\xi \xi}(x, \xi) \lambda, \lambda\right\rangle \geq c_{0} \nu\left(1+|\xi|^{2}\right)^{\frac{p-2}{2}}|\lambda|^{2} \quad \forall \lambda \in \mathbb{R}^{n N} .
$$

where the constant $\nu$ is the same constant appearing in (H2).

The next result about finite difference operator is a kind of integral version of Lagrange theorem.

Lemma 2.2. If $0<\rho<R,|h|<\frac{R-\rho}{2}, 1 \leq p<+\infty, s \in\{1, \ldots, n\}$ and $F, D_{s} F \in L^{p}\left(B_{R}\right)$ then

$$
\int_{B_{\rho}}\left|\tau_{s, h} F(x)\right|^{p} \mathrm{~d} x \leq|h|^{p} \int_{B_{R}}\left|D_{s} F(x)\right|^{p} \mathrm{~d} x .
$$

Moreover

$$
\int_{B_{\rho}}\left|F\left(x+h e_{s}\right)\right|^{p} \mathrm{~d} x \leq c(n, p) \int_{B_{R}}|F(x)|^{p} \mathrm{~d} x .
$$

The following result is standard if $p \geq 2$ and can be inferred from [2] (Lem. 2.2) in the case $1<p<2$.

Lemma 2.3. For every $p>1$ and $G: B_{R} \rightarrow \mathbb{R}^{k}$ there exists a positive constant $c \equiv c(k, p)$ such that

$$
\left|\tau_{s, h}\left(\left(1+|G(x)|^{2}\right)^{(p-2) / 4} G(x)\right)\right|^{2} \leq c\left(1+|G(x)|^{2}+\left|G\left(x+h e_{s}\right)\right|^{2}\right)^{(p-2) / 2}\left|\tau_{s, h} G(x)\right|^{2}
$$

for every $x \in B_{\rho}$, with $|h|<\frac{R-\rho}{2}$ and every $s \in\{1, \ldots, n\}$.

Now we recall the fundamental embedding properties for fractional order Sobolev spaces. (For the proof see, for example, [3].)

Lemma 2.4. If $F: \mathbb{R}^{n} \rightarrow \mathbb{R}^{N}, F \in L^{2}\left(B_{R}\right)$ and for some $\rho \in(0, R), \beta \in(0,1], M>0$,

$$
\sum_{s=1}^{n} \int_{B_{\rho}}\left|\tau_{s, h} F(x)\right|^{2} \mathrm{~d} x \leq M^{2}|h|^{2 \beta}
$$

for every $h$ with $|h|<\frac{R-\rho}{2}$, then $F \in W^{k, 2}\left(B_{\rho} ; \mathbb{R}^{N}\right) \cap L^{\frac{2 n}{n-2 k}}\left(B_{\rho} ; \mathbb{R}^{N}\right)$ for every $k \in(0, \beta)$ and

$$
\|F\|_{L^{\frac{2 n}{n-2 k}\left(B_{\rho}\right)}} \leq c\left(M+\|F\|_{L^{2}\left(B_{R}\right)}\right)
$$

with $c \equiv c(n, N, R, \rho, \beta, k)$.

The next result is in fact a reformulation of the previous lemma. We shall also need the following version of embedding lemma since the cases $1<p<2$ and $p \geq 2$ have different technical difficulties.

Lemma 2.5. If $F: \mathbb{R}^{n} \rightarrow \mathbb{R}^{N}, F \in L^{p}\left(B_{R}\right)$ with $1<p<+\infty$ and for some $\rho \in(0, R), \beta \in(0,1], M>0$,

$$
\sum_{s=1}^{n} \int_{B_{\rho}}\left|\tau_{s, h} F(x)\right|^{p} \mathrm{~d} x \leq M^{p}|h|^{p \beta}
$$

for every $h$ with $|h|<\frac{R-\rho}{2}$, then $F \in W^{k, p}\left(B_{\rho} ; \mathbb{R}^{N}\right) \cap L^{\frac{n p}{n-k p}}\left(B_{\rho} ; \mathbb{R}^{N}\right)$ for every $k \in(0, \beta)$ and

$$
\|F\|_{L^{\frac{n p}{n-k p}}\left(B_{\rho}\right)} \leq c\left(M+\|F\|_{L^{p}\left(B_{R}\right)}\right)
$$

with $c \equiv c(n, N, R, \rho, \beta, k)$. 
Now let us recall that the singular set $\Sigma$ of a local minimizer $u$ of the functional $\mathcal{F}$ is included in the set of non-Lebesgue points of $D u$. Therefore the estimate for the Hausdorff dimension of $\Sigma$ is an immediate corollary of the regularity Theorems 1.1 and 1.2 through the application of the following proposition that can be found, for example, in [20] (see also Sect. 4 in [21] for a simple proof).

Lemma 2.6. Let $v \in W^{\theta, p}\left(\Omega, \mathbb{R}^{N}\right)$ where $\theta \in(0,1), p>1$ and set

$$
\begin{gathered}
A:=\left\{x \in \Omega: \limsup _{\rho \rightarrow 0^{+}} f_{B(x, \rho)}\left|v(y)-(v)_{x, \rho}\right|^{p} \mathrm{~d} y>0\right\}, \\
B:=\left\{\underset{\rho \rightarrow 0^{+}}{x \in \Omega: \limsup _{j, \rho}\left|(v)_{x, 1}\right|+\infty} .\right.
\end{gathered}
$$

Then

$$
\operatorname{dim}_{\mathcal{H}}(A) \leq n-\theta p \quad \text { and } \quad \operatorname{dim}_{\mathcal{H}}(B) \leq n-\theta p
$$

\section{A PRIORI ESTIMATES}

In this section we prove the estimates (1.6) and (1.9) assuming that $f$ satisfies the growth assumption (H1), the Hölder condition $(H 3)$ and $f(\cdot, \xi) \in C^{2}$ for every $\xi \in \mathbb{R}^{n N}$. Recall (see (2.1)) that under this last assumption $(H 2)$ is equivalent to the ellipticity condition (1.2) with the same $\nu$ appearing in $(H 2)$. Then in the next section we use the fundamental approximation procedure of [14], to prove Theorems 1.1 and 1.2 . In any case we explicitly point out that in this section we establish the estimates (1.6) and (1.9) independently of the $C^{2}$ norm of $f$.

Now we observe that the convexity assumption $(H 2)$ together with $(H 1)$ implies the estimate

$$
\left|f_{\xi}(x, \xi)\right| \leq c\left(1+|\xi|^{2}\right)^{\frac{p-1}{2}} \quad \forall(x, \xi) \in \Omega \times \mathbb{R}^{n N}
$$

where $c \equiv c(n, N, p, L)$.

We start proving (1.6).

Lemma 3.1. Suppose $f$ satisfies $(H 1)$, (H3) for a $p \geq 2$ and $f(\cdot, \xi) \in C^{2}$ for every $\xi \in \mathbb{R}^{n N}$. If $u \in W^{1, p}\left(\Omega ; \mathbb{R}^{N}\right)$ is a local minimizer of $\mathcal{F}$ then the estimate (1.6) holds.

Proof. We assumed $f(\cdot, \xi) \in C^{2}$ so $f$ satisfies the ellipticity condition (1.2) by Lemma 2.1. Let $u \in W^{1, p}\left(\Omega ; \mathbb{R}^{N}\right)$ be a local minimizer of the functional $\mathcal{F}$ and let us take $0<R<1$ such that $B_{2 R} \subset \subset \Omega$; then $u$ is a solution of the Euler system

$$
\int_{\Omega} f_{\xi}(x, D u) D \varphi \mathrm{d} x=0
$$

for every $\varphi \in W^{1, p}\left(\Omega ; \mathbb{R}^{N}\right)$ such that $\operatorname{supp} \varphi \subset \subset \Omega$.

Let $\eta$ be a cut-off function in $C_{0}^{1}\left(B_{3 R / 2}\right)$ with $0 \leq \eta \leq 1, \eta \equiv 1$ on $B_{R}$ and $|D \eta|<c / R$. Let us consider the function $\varphi=\eta^{2}(x) \tau_{s,-h}\left(\tau_{s, h} u\right.$ ) with $s$ fixed in $\{1, \ldots, n\}$ (which from now on we shall omit for the sake of simplicity) and $|h|<R / 10$. Substituting in (3.2) the function $\varphi$ we get

$$
\int_{B_{2 R}} \eta^{2}(x) f_{\xi}(x, D u) D\left(\tau_{-h}\left(\tau_{h} u\right)\right) \mathrm{d} x=-2 \int_{B_{2 R}} f_{\xi}(x, D u) \eta(x) D \eta \otimes \tau_{-h}\left(\tau_{h} u\right) \mathrm{d} x
$$


and thanks to $(d 1)$ and $(d 2)$ of Proposition 2.1 we get

$$
\begin{aligned}
\int_{B_{2 R}} & \eta^{2}\left(x+h e_{s}\right)\left[f_{\xi}\left(x+h e_{s}, D u\left(x+h e_{s}\right)\right)-f_{\xi}\left(x+h e_{s}, D u(x)\right)\right] D\left(\tau_{h} u\right) \mathrm{d} x \\
& +\int_{B_{2 R}} \eta^{2}\left(x+h e_{s}\right)\left[f_{\xi}\left(x+h e_{s}, D u(x)\right)-f_{\xi}(x, D u(x))\right] D\left(\tau_{h} u\right) \mathrm{d} x \\
& +\int_{B_{2 R}}\left[\eta^{2}\left(x+h e_{s}\right)-\eta^{2}(x)\right] f_{\xi}(x, D u) D\left(\tau_{h} u\right) \mathrm{d} x \\
& =2 \int_{B_{2 R}} f_{\xi}(x, D u) \eta(x) D \eta \otimes \tau_{-h}\left(\tau_{h} u\right) \mathrm{d} x .
\end{aligned}
$$

Assumption (H3) and inequality (3.1) yield

$$
\begin{aligned}
& \int_{B_{2 R}} \eta^{2}\left(x+h e_{s}\right)\left[f_{\xi}\left(x+h e_{s}, D u\left(x+h e_{s}\right)\right)-f_{\xi}\left(x+h e_{s}, D u(x)\right)\right] D\left(\tau_{h} u\right) \mathrm{d} x \\
& \leq c|h|^{\alpha} \int_{B_{2 R}} \eta^{2}\left(x+h e_{s}\right)\left|D\left(\tau_{h} u\right)\right|\left(1+|D u|^{2}\right)^{\frac{p-1}{2}} \mathrm{~d} x \\
&+c \int_{B_{2 R}}\left|\eta^{2}\left(x+h e_{s}\right)-\eta^{2}(x)\right|\left|D\left(\tau_{h} u\right)\right|\left(1+|D u|^{2}\right)^{\frac{p-1}{2}} \mathrm{~d} x \\
&+c \int_{B_{2 R}} \eta(x)|D \eta|\left|\tau_{-h}\left(\tau_{h} u\right)\right|\left(1+|D u|^{2}\right)^{\frac{p-1}{2}} \mathrm{~d} x
\end{aligned}
$$

with $c \equiv c(n, N, p, L)$.

Now we can use the ellipticity condition (1.2) in the left hand side of (3.3) as follows

$$
\begin{aligned}
& \int_{B_{2 R}} \eta^{2}\left(x+h e_{s}\right)\left(1+|D u(x)|^{2}+\left|D u\left(x+h e_{s}\right)\right|^{2}\right)^{\frac{p-2}{2}}\left|\tau_{h} D u\right|^{2} \mathrm{~d} x \\
& \quad \leq \int_{B_{2 R}} \int_{0}^{1}\left[f_{\xi \xi}\left(x+h e_{s}, D u+t \tau_{h} D u\right)\right] \eta^{2}\left(x+h e_{s}\right) D\left(\tau_{h} u\right) D\left(\tau_{h} u\right) \mathrm{d} t \mathrm{~d} x
\end{aligned}
$$

and, since

$$
\left|\tau_{h} D u\right|^{p}=\left|\tau_{h} D u\right|^{p-2}\left|\tau_{h} D u\right|^{2} \leq c(n, p)\left(1+|D u(x)|^{2}+\left|D u\left(x+h e_{s}\right)\right|^{2}\right)^{\frac{p-2}{2}}\left|\tau_{h} D u\right|^{2}
$$

and $p \geq 2$, we get the estimate

$$
\begin{aligned}
\int_{B_{2 R}} \eta^{2}\left(x+h e_{s}\right)\left|\tau_{h} D u\right|^{p} \mathrm{~d} x \leq & c|h|^{\alpha} \int_{B_{2 R}} \eta^{2}\left(x+h e_{s}\right)\left|\tau_{h} D u\right|\left(1+|D u|^{2}\right)^{\frac{p-1}{2}} \mathrm{~d} x \\
& +c \int_{B_{2 R}}\left|\eta^{2}\left(x+h e_{s}\right)-\eta^{2}(x)\right|\left|\tau_{h} D u\right|\left(1+|D u|^{2}\right)^{\frac{p-1}{2}} \mathrm{~d} x \\
& +c \int_{B_{2 R}} \eta(x)|D \eta|\left|\tau_{-h}\left(\tau_{h} u\right)\right|\left(1+|D u|^{2}\right)^{\frac{p-1}{2}} \mathrm{~d} x \\
:= & (I)+(I I)+(I I I),
\end{aligned}
$$

with $c \equiv c(n, N, p, L, \nu)$. We have to estimate the integrals appearing in the right hand side of (3.4). In what follows $\epsilon$ is a real number such that $0<\epsilon<1$ to be chosen later. 
Let us begin from $(I)$. We can apply Young's inequality with the exponents $p$ and $q \equiv \frac{p}{p-1}$ so we have

$$
(I) \leq c|h|^{q \alpha} \int_{B_{2 R}} \eta^{2}\left(x+h e_{s}\right)\left(1+|D u(x)|^{2}\right)^{\frac{p}{2}} \mathrm{~d} x+c \epsilon \int_{B_{2 R}} \eta^{2}\left(x+h e_{s}\right)\left|\tau_{h} D u\right|^{p} \mathrm{~d} x
$$

where $c \equiv c(n, N, p, L, \nu)$.

Let us estimate $(I I)$; we can apply Lagrange's theorem to estimate $\left|\eta^{2}\left(x+h e_{s}\right)-\eta^{2}(x)\right|$, the assumptions on $|D \eta|$ and again Young's inequality with exponents $p$ and $q$ obtaining

$$
\begin{aligned}
(I I) & \leq c \frac{|h|}{R} \int_{B_{2 R}}\left|\eta\left(x+h e_{s}\right)+\eta(x)\right|\left(1+|D u(x)|^{2}\right)^{\frac{p-1}{2}}\left|\tau_{h} D u\right| \mathrm{d} x \\
& \leq c \frac{|h|^{q}}{R^{q}} \int_{B_{2 R}}\left(1+|D u(x)|^{2}\right)^{\frac{p}{2}} \mathrm{~d} x+c \epsilon \int_{B_{2 R}}\left|\eta^{p}\left(x+h e_{s}\right)+\eta^{p}(x)\right|\left|\tau_{h} D u\right|^{p} \mathrm{~d} x \\
& \leq c \frac{|h|^{q}}{R^{q}} \int_{B_{2 R}}\left(1+|D u(x)|^{2}\right)^{\frac{p}{2}} \mathrm{~d} x+c \epsilon \int_{B_{2 R}} \eta^{p}\left(x+h e_{s}\right)\left|\tau_{h} D u\right|^{p} \mathrm{~d} x+c \epsilon \int_{B_{2 R}} \eta^{p}(x)\left|\tau_{h} D u\right|^{p} \mathrm{~d} x \\
& \leq c \frac{|h|^{q}}{R^{q}} \int_{B_{2 R}}\left(1+|D u(x)|^{2}\right)^{\frac{p}{2}} \mathrm{~d} x+c \epsilon \int_{B_{2 R}} \eta^{2}\left(x+h e_{s}\right)\left|\tau_{h} D u\right|^{p} \mathrm{~d} x+c \epsilon \int_{B_{2 R}} \eta^{2}(x)\left|\tau_{h} D u\right|^{p} \mathrm{~d} x,
\end{aligned}
$$

where we used the assumptions $p \geq 2$ and $0 \leq \eta \leq 1$ to get the last estimate.

To estimate $(I I I)$ we use, once again, Young's inequality and the properties of $\eta$ obtaining

$$
(I I I) \leq c \frac{|h|^{q}}{R^{q}} \int_{B_{2 R}}\left(1+|D u(x)|^{2}\right)^{\frac{p}{2}} \mathrm{~d} x+\frac{c \epsilon}{|h|^{p}} \int_{B_{2 R}} \eta^{p}(x)\left|\tau_{-h}\left(\tau_{h} u\right)\right|^{p} \mathrm{~d} x .
$$

Now using the definition of $\tau_{h} u$ we can write the last integral in (3.5) as follows

$$
\begin{aligned}
& \frac{c \epsilon}{|h|^{p}} \int_{B_{2 R}}\left|\eta\left(x-h e_{s}\right)\left(\tau_{h} u\right)\left(x-h e_{s}\right)-\eta(x)\left(\tau_{h} u\right)(x)+\left(\eta(x)-\eta\left(x-h e_{s}\right)\right)\left(\tau_{h} u\right)\left(x-h e_{s}\right)\right|^{p} \mathrm{~d} x \\
& \quad \leq \frac{c \epsilon}{|h|^{p}} \int_{B_{2 R}}\left|\tau_{-h}\left(\eta \tau_{h} u\right)(x)\right|^{p} \mathrm{~d} x+\frac{c}{|h|^{p}} \int_{B_{2 R}}\left|\eta(x)-\eta\left(x-h e_{s}\right)\right|^{p}\left|\left(\tau_{h} u\right)\left(x-h e_{s}\right)\right|^{p} \mathrm{~d} x \\
& \quad \leq c \epsilon \int_{B_{2 R}}\left|D\left(\eta \tau_{h} u\right)(x)\right|^{p} \mathrm{~d} x+\frac{c \epsilon}{R^{p}} \int_{B_{2 R}}\left|\left(\tau_{h} u\right)\left(x-h e_{s}\right)\right|^{p} \mathrm{~d} x,
\end{aligned}
$$

where we used Lemma 2.2 and Lagrange's theorem to get the last estimate.

Recalling how we chose $\eta$ and $|h|$ at the beginning we can estimate the last sum in (3.6) with

$$
\begin{aligned}
& c \epsilon \int_{B_{2 R}}\left|D\left(\eta \tau_{h} u\right)(x)\right|^{p} \mathrm{~d} x+\frac{c \epsilon}{R^{p}} \int_{B_{2 R}}\left|\left(\tau_{h} u\right)(x)\right|^{p} \mathrm{~d} x \\
& \quad \leq c \epsilon \int_{B_{2 R}} \eta^{p}(x)\left|\tau_{h}(D u)(x)\right|^{p} \mathrm{~d} x+c \epsilon \int_{B_{2 R}}|D \eta|^{p}\left|\left(\tau_{h} u\right)(x)\right|^{p} \mathrm{~d} x+\frac{c \epsilon}{R^{p}} \int_{B_{2 R}}\left|\left(\tau_{h} u\right)(x)\right|^{p} \mathrm{~d} x \\
& \quad \leq c \epsilon \int_{B_{2 R}} \eta^{2}(x)\left|\tau_{h}(D u)(x)\right|^{p} \mathrm{~d} x+\frac{c \epsilon}{R^{p}} \int_{B_{2 R}}\left|\left(\tau_{h} u\right)(x)\right|^{p} \mathrm{~d} x,
\end{aligned}
$$

where we used the assumptions on $p$ and $\eta$ again. So we have

$$
(I I I) \leq c \frac{|h|^{q}}{R^{q}} \int_{B_{2 R}}\left(1+|D u(x)|^{2}\right)^{\frac{p}{2}} \mathrm{~d} x+c \epsilon \int_{B_{2 R}} \eta^{2}(x)\left|\tau_{h}(D u)(x)\right|^{p} \mathrm{~d} x+\frac{c \epsilon}{R^{p}} \int_{B_{2 R}}\left|\left(\tau_{h} u\right)(x)\right|^{p} \mathrm{~d} x .
$$


Since $\tau_{h} D u(x)=D u\left(x+h e_{s}\right)-D u(x)$ and noting that

$$
\begin{aligned}
c \epsilon \int_{B_{2 R}} \eta^{p}(x)\left|\tau_{h} D u\right|^{p} \mathrm{~d} x & \leq c \int_{B_{2 R}}\left|\eta(x)-\eta\left(x+h e_{s}\right)\right|^{p}\left|\tau_{h}(D u)(x)\right|^{p} \mathrm{~d} x+c \epsilon \int_{B_{2 R}} \eta^{p}\left(x+h e_{s}\right)\left|\tau_{h} D u(x)\right|^{p} \mathrm{~d} x \\
& \leq c \frac{|h|^{p}}{R^{p}} \int_{B_{2 R}}\left|\tau_{h} D u(x)\right|^{p} \mathrm{~d} x+c \epsilon \int_{B_{2 R}} \eta^{2}\left(x+h e_{s}\right)\left|\tau_{h} D u\right|^{p} \mathrm{~d} x \\
& \leq c \frac{|h|^{p}}{R^{p}} \int_{B_{2 R}}\left(1+|D u|^{2}\right)^{\frac{p}{2}} \mathrm{~d} x+c \epsilon \int_{B_{2 R}} \eta^{2}\left(x+h e_{s}\right)\left|\tau_{h} D u\right|^{p} \mathrm{~d} x
\end{aligned}
$$

we obtain

$$
(I I I) \leq c \frac{|h|^{p}}{R^{p}} \int_{B_{2 R}}\left(1+|D u(x)|^{2}\right)^{\frac{p}{2}} \mathrm{~d} x+c \epsilon \int_{B_{2 R}} \eta^{2}\left(x+h e_{s}\right)\left|\tau_{h} D u\right|^{p} \mathrm{~d} x+c \frac{|h|^{q}}{R^{q}} \int_{B_{2 R}}\left(1+|D u(x)|^{2}\right)^{\frac{p}{2}} \mathrm{~d} x .
$$

Collecting the estimates for $(I),(I I)$ and $(I I I)$ we get

$$
\begin{aligned}
\int_{B_{2 R}} \eta^{2}\left(x+h e_{s}\right)\left|\tau_{h} D u\right|^{p} \mathrm{~d} x \leq & c \epsilon \int_{B_{2 R}} \eta^{2}\left(x+h e_{s}\right)\left|\tau_{h} D u\right|^{p} \mathrm{~d} x+c \frac{|h|^{p}}{R^{p}} \int_{B_{2 R}}\left(1+|D u(x)|^{2}\right)^{\frac{p}{2}} \mathrm{~d} x \\
& +c|h|^{q \alpha} \int_{B_{2 R}}\left(1+|D u(x)|^{2}\right)^{\frac{p}{2}} \mathrm{~d} x+c \frac{|h|^{q}}{R^{q}} \int_{B_{2 R}}\left(1+|D u(x)|^{2}\right)^{\frac{p}{2}} \mathrm{~d} x .
\end{aligned}
$$

Now choosing $\epsilon>0$ small enough we get

$$
\begin{aligned}
\int_{B_{2 R}} \eta^{2}\left(x+h e_{s}\right)\left|\tau_{h} D u\right|^{p} \mathrm{~d} x \leq & c|h|^{q \alpha} \int_{B_{2 R}}\left(1+|D u(x)|^{2}\right)^{\frac{p}{2}} \mathrm{~d} x+c \frac{|h|^{q}}{R^{q}} \int_{B_{2 R}}\left(1+|D u(x)|^{2}\right)^{\frac{p}{2}} \mathrm{~d} x \\
& +c \frac{|h|^{p}}{R^{p}} \int_{B_{2 R}}\left(1+|D u(x)|^{2}\right)^{\frac{p}{2}} \mathrm{~d} x
\end{aligned}
$$

but since $q \alpha<q, q \leq 2$ and recalling that $R<1$ the following estimate easily follows

$$
\int_{B_{2 R}} \eta^{2}\left(x+h e_{s}\right)\left|\tau_{h} D u\right|^{p} \mathrm{~d} x \leq c|h|^{p\left(\frac{\alpha}{p-1}\right)} \int_{B_{2 R}}\left(1+|D u(x)|^{p}\right) \mathrm{d} x
$$

with $c \equiv c(n, N, L, \nu, p, R)$. We can conclude applying Lemma 2.5 and performing a standard covering procedure.

Now we prove (1.9) again under the $C^{2}$ regularity assumption on the integrand $f$.

Lemma 3.2. Suppose $f$ satisfies $(H 1)$, (H3), for $a 1<p<2$ and $f(\cdot, \xi) \in C^{2}$ for every $\xi \in \mathbb{R}^{n N}$. If $u \in W^{1, p}\left(\Omega, \mathbb{R}^{N}\right)$ is a local minimizer of $\mathcal{F}$ then the estimate (1.9) holds.

Proof. Let $\eta$ be a cut-off function in $C_{0}^{1}\left(B_{3 R / 2}\right)$ with $0 \leq \eta \leq 1, \eta \equiv 1$ on $B_{R}$ and $|D \eta|<c / R$. Let us consider the function $\varphi=\eta^{2}(x) \tau_{s,-h}\left(\tau_{s, h} u\right)$ with $s$ fixed in $\{1, \ldots, n\}$ (which from now on we shall omit for the sake of simplicity) and $|h|<R / 10$. Substituting in (3.2) the function $\varphi$ and arguing as in the first part of the proof 
of Lemma 3.1 we get the estimate

$$
\begin{aligned}
& \int_{B_{2 R}} \eta^{2}\left(x+h e_{s}\right)\left(1+|D u(x)|^{2}+\left|D u\left(x+h e_{s}\right)\right|^{2}\right)^{\frac{p-2}{2}}\left|\tau_{h} D u\right|^{2} \mathrm{~d} x \\
& \leq c|h|^{\alpha} \int_{B_{2 R}} \eta^{2}\left(x+h e_{s}\right)\left|\tau_{h} D u\right|\left(1+|D u|^{2}\right)^{\frac{p-1}{2}} \mathrm{~d} x \\
& \quad+c \int_{B_{2 R}}\left|\eta^{2}\left(x+h e_{s}\right)-\eta^{2}(x)\right|\left|\tau_{h} D u\right|\left(1+|D u|^{2}\right)^{\frac{p-1}{2}} \mathrm{~d} x \\
& \quad+c \int_{B_{2 R}} \eta(x)|D \eta|\left|\tau_{-h}\left(\tau_{h} u\right)\right|\left(1+|D u|^{2}\right)^{\frac{p-1}{2}} \mathrm{~d} x \\
& :=(I)+(I I)+(I I I),
\end{aligned}
$$

with $c \equiv c(n, N, p, L, \nu)$. We have to estimate the integrals appearing in the right hand side of (3.8). In what follows $\epsilon$ is a real number such that $0<\epsilon<1$ to be chosen later.

Let us begin from $(I)$. Observing that

$$
\frac{p-1}{2}=\frac{p-2}{4}+\frac{p}{4}
$$

we can apply Young's inequality with the exponent 2, so we have

$$
\begin{aligned}
(I) \leq & c|h|^{\alpha} \int_{B_{2 R}} \eta^{2}\left(x+h e_{s}\right)\left(1+|D u(x)|^{2}+\left|D u\left(x+h e_{s}\right)\right|^{2}\right)^{\frac{p-1}{2}}\left|\tau_{h} D u\right| \mathrm{d} x \\
\leq & c \epsilon|h|^{2 \alpha} \int_{B_{2 R}} \eta^{2}\left(x+h e_{s}\right)\left(1+|D u(x)|^{2}+\left|D u\left(x+h e_{s}\right)\right|^{2}\right)^{\frac{p}{2}} \mathrm{~d} x \\
& +\epsilon \int_{B_{2 R}} \eta^{2}\left(x+h e_{s}\right)\left(1+|D u(x)|^{2}+\left|D u\left(x+h e_{s}\right)\right|^{2}\right)^{\frac{p-2}{2}}\left|\tau_{h} D u\right|^{2} \mathrm{~d} x \\
\leq & c \epsilon|h|^{2 \alpha} \int_{B_{2 R}}\left(1+|D u(x)|^{2}\right)^{\frac{p}{2}} \mathrm{~d} x \\
& +\epsilon \int_{B_{2 R}} \eta^{2}\left(x+h e_{s}\right)\left(1+|D u(x)|^{2}+\left|D u\left(x+h e_{s}\right)\right|^{2}\right)^{\frac{p-2}{2}}\left|\tau_{h} D u\right|^{2} \mathrm{~d} x
\end{aligned}
$$

with $c \equiv c(n, N, p, L, \nu)$, where in the last inequality we used Lemma 2.2.

Now let us estimate $(I I)$. We can apply Lagrange's theorem to estimate $\left|\eta^{2}\left(x+h e_{s}\right)-\eta^{2}(x)\right|$, the assumption on $|D \eta|$ and again Young's inequality with exponent 2 obtaining

$$
\begin{aligned}
(I I) \leq & c \frac{|h|}{R} \int_{B_{2 R}}\left|\eta\left(x+h e_{s}\right)+\eta(x)\right|\left(1+|D u(x)|^{2}+\left|D u\left(x+h e_{s}\right)\right|^{2}\right)^{\frac{p-1}{2}}\left|\tau_{h} D u\right| \mathrm{d} x \\
\leq & c \epsilon \frac{|h|^{2}}{R^{2}} \int_{B_{2 R}}\left(1+|D u(x)|^{2}+\left|D u\left(x+h e_{s}\right)\right|^{2}\right)^{\frac{p}{2}} \mathrm{~d} x \\
& +\epsilon \int_{B_{2 R}}\left|\eta^{2}\left(x+h e_{s}\right)+\eta^{2}(x)\right|\left(1+|D u(x)|^{2}+\left|D u\left(x+h e_{s}\right)\right|^{2}\right)^{\frac{p-2}{2}}\left|\tau_{h} D u\right|^{2} \mathrm{~d} x \\
\leq & c \epsilon \frac{|h|^{2}}{R^{2}} \int_{B_{2 R}}\left(1+|D u(x)|^{2}\right)^{\frac{p}{2}} \mathrm{~d} x+\epsilon \int_{B_{2 R}} \eta^{2}\left(x+h e_{s}\right)\left(1+|D u(x)|^{2}+\left|D u\left(x+h e_{s}\right)\right|^{2}\right)^{\frac{p-2}{2}}\left|\tau_{h} D u\right|^{2} \mathrm{~d} x \\
& +\epsilon \int_{B_{2 R}} \eta^{2}(x)\left(1+|D u(x)|^{2}+\left|D u\left(x+h e_{s}\right)\right|^{2}\right)^{\frac{p-2}{2}}\left|\tau_{h} D u\right|^{2} \mathrm{~d} x
\end{aligned}
$$

where we used Lemma 2.2. 
To estimate $(I I I)$ we use Hölder's inequality, the definition of $\tau_{h} u$ and the properties of $\eta$ and $h$ obtaining

$$
\begin{aligned}
(I I I) & \leq \frac{c}{R}\left(\int_{B_{2 R}}\left(1+|D u(x)|^{2}+\left|D u\left(x+h e_{s}\right)\right|^{2}\right)^{\frac{p}{2}}\right)^{1-\frac{1}{p}}\left(\int_{B_{2 R}}\left|\tau_{-h}\left(\tau_{h} u\right)\right|^{p} \mathrm{~d} x\right)^{\frac{1}{p}} \\
& \leq|h| \frac{c}{R} \int_{B_{2 R}}\left(1+|D u(x)|^{p}\right) \mathrm{d} x,
\end{aligned}
$$

where we also used Lemma 2.2. Now set

$$
W_{h}=W_{h}(D u)=1+|D u(x)|^{2}+\left|D u\left(x+h e_{s}\right)\right|^{2}
$$

and, since $\tau_{h} D u(x)=D u\left(x+h e_{s}\right)-D u(x)$, we get

$$
\begin{aligned}
c \int_{B_{2 R}} W_{h}^{\frac{p-2}{2}} \eta^{2}(x)\left|\tau_{h} D u\right|^{2} \mathrm{~d} x \leq & c \epsilon \int_{B_{2 R}} W_{h}^{\frac{p-2}{2}}\left|\eta(x)-\eta\left(x+h e_{s}\right)\right|^{2}\left|\tau_{h}(D u)(x)\right|^{2} \mathrm{~d} x \\
& +c \epsilon \int_{B_{2 R}} W_{h}^{\frac{p-2}{2}} \eta^{2}\left(x+h e_{s}\right)\left|\tau_{h}(D u)(x)\right|^{2} \mathrm{~d} x \\
\leq & c \frac{|h|^{2}}{R^{2}} \int_{B_{2 R}} W_{h}^{\frac{p-2}{2}}\left|\tau_{h} D u(x)\right|^{2} \mathrm{~d} x+\epsilon \int_{B_{2 R}} W_{h}^{\frac{p-2}{2}} \eta^{2}\left(x+h e_{s}\right)\left|\tau_{h} D u\right|^{2} \mathrm{~d} x \\
\leq & c \frac{|h|^{2}}{R^{2}} \int_{B_{2 R}} W_{h}^{\frac{p-2}{2}}|D u(x)|^{2} \mathrm{~d} x+c \epsilon \int_{B_{2 R}} W_{h}^{\frac{p-2}{2}} \eta^{2}\left(x+h e_{s}\right)\left|\tau_{h} D u\right|^{2} \mathrm{~d} x \\
\leq & c \frac{|h|^{2}}{R^{2}} \int_{B_{2 R}}\left(1+|D u(x)|^{2}\right)^{\frac{p}{2}} \mathrm{~d} x+c \epsilon \int_{B_{2 R}} W_{h}^{\frac{p-2}{2}} \eta^{2}\left(x+h e_{s}\right)\left|\tau_{h} D u\right|^{2} \mathrm{~d} x .
\end{aligned}
$$

Collecting the estimates for $(I),(I I)$ and $(I I I)$, we get

$$
\begin{aligned}
& \int_{B_{2 R}} \eta^{2}\left(x+h e_{s}\right)\left(1+|D u(x)|^{2}+\left|D u\left(x+h e_{s}\right)\right|^{2}\right)^{\frac{p-2}{2}}\left|\tau_{h} D u\right|^{2} \mathrm{~d} x \\
& \leq c \epsilon \int_{B_{2 R}} W_{h}^{\frac{p-2}{2}} \eta^{2}\left(x+h e_{s}\right)\left|\tau_{h} D u\right|^{2} \mathrm{~d} x+c \frac{|h|^{2}}{R^{2}} \int_{B_{2 R}}\left(1+|D u(x)|^{p}\right) \mathrm{d} x \\
& \quad+c \epsilon|h|^{2 \alpha} \int_{B_{2 R}}\left(1+|D u(x)|^{p}\right) \mathrm{d} x+|h| \frac{c}{R} \int_{B_{2 R}}\left(1+|D u(x)|^{p}\right) \mathrm{d} x .
\end{aligned}
$$

From this estimate, choosing $\epsilon>0$ small enough, we get

$$
\begin{aligned}
& \int_{B_{2 R}} \eta^{2}\left(x+h e_{s}\right)\left(1+|D u(x)|^{2}+\left|D u\left(x+h e_{s}\right)\right|^{2}\right)^{\frac{p-2}{2}}\left|\tau_{h} D u\right|^{2} \mathrm{~d} x \\
& \quad \leq c \frac{|h|^{2}}{R^{2}} \int_{B_{2 R}}\left(1+|D u(x)|^{p}\right) \mathrm{d} x+c \epsilon|h|^{2 \alpha} \int_{B_{2 R}}\left(1+|D u(x)|^{p}\right) \mathrm{d} x+|h| \frac{c}{R} \int_{B_{2 R}}\left(1+|D u(x)|^{p}\right) \mathrm{d} x,
\end{aligned}
$$

but since $2 \alpha<2$ and $R<1$ the following estimate easily follows

$$
\begin{aligned}
& \int_{B_{2 R}} \eta^{2}\left(x+h e_{s}\right)\left(1+|D u(x)|^{2}+\left|D u\left(x+h e_{s}\right)\right|^{2}\right)^{\frac{p-2}{2}}\left|\tau_{h} D u\right|^{2} \mathrm{~d} x \\
& \leq c|h|^{2 \alpha} \int_{B_{2 R}}\left(1+|D u(x)|^{p}\right) \mathrm{d} x+c|h| \int_{B_{2 R}}\left(1+|D u(x)|^{p}\right) \mathrm{d} x,
\end{aligned}
$$

with $c \equiv c(n, N, L, \nu, p, R)$. 
Now if $2 \alpha<1$, that is $\alpha<\frac{1}{2}$, we have

$$
\int_{B_{2 R}} \eta^{2}\left(x+h e_{s}\right)\left(1+|D u(x)|^{2}+\left|D u\left(x+h e_{s}\right)\right|^{2}\right)^{\frac{p-2}{2}}\left|\tau_{h} D u\right|^{2} \mathrm{~d} x \leq c|h|^{2 \alpha} \int_{B_{2 R}}\left(1+|D u(x)|^{p}\right) \mathrm{d} x
$$

while, if $\alpha \geq \frac{1}{2}$ we have

$$
\int_{B_{2 R}} \eta^{2}\left(x+h e_{s}\right)\left(1+|D u(x)|^{2}+\left|D u\left(x+h e_{s}\right)\right|^{2}\right)^{\frac{p-2}{2}}\left|\tau_{h} D u\right|^{2} \mathrm{~d} x \leq c|h| \int_{B_{2 R}}\left(1+|D u(x)|^{p}\right) \mathrm{d} x .
$$

We can get the final estimate applying Lemma 2.3 which yields

$$
\int_{B_{R}}\left|\tau_{s, h}\left(\left(1+|D u(x)|^{2}\right)^{(p-2) / 4} D u(x)\right)\right|^{2} \mathrm{~d} x \leq c|h|^{\beta} \int_{B_{2 R}}\left(1+|D u(x)|^{p}\right) \mathrm{d} x
$$

where

$$
\begin{array}{ccc}
\beta=2 \alpha \quad \text { if } & \alpha<\frac{1}{2}, \\
\beta=1 \quad \text { if } & \alpha \geq \frac{1}{2} .
\end{array}
$$

Now we can conclude applying Lemma 2.4 and performing a standard covering procedure.

\section{THE APPROXIMATION}

We need now the following fundamental result that can be obtained with a procedure first introduced in $[14,15]$ and then developed in $[7,10]$, that plays a key role in the completion of the proof of our theorems.

Lemma 4.1. Let us suppose that the function $f$ satisfies assumptions $(H 1),(H 2)$ and $(H 3)$. Then there exist a sequence $f_{h}(x, \cdot) \in C^{2}\left(\mathbb{R}^{n N}\right)$ and a constant $c>1$ independent of $h$ such that

$$
\begin{array}{cc}
\text { (i) } \quad \frac{1}{c}\left(1+\frac{1}{h^{2}}+|\xi|^{2}\right)^{\frac{p}{2}} \leq f_{h}(x, \xi) \leq c L\left(1+\frac{1}{h^{2}}+|\xi|^{2}\right)^{\frac{p}{2}} & \forall x \in \Omega, \forall \lambda, \xi \in \mathbb{R}^{n N} ; \\
\frac{\nu}{c}|\lambda|^{2}\left(1+|\xi|^{2}\right)^{\frac{p-2}{2}} \leq\left\langle D_{i j}^{2} f_{h}(x, \xi) \lambda_{i} \lambda_{j}\right\rangle & \forall x \in \Omega, \forall \lambda, \xi \in \mathbb{R}^{n N} ; \\
\text { (ii) } \quad\left|D_{\xi} f_{h}\left(x_{1}, \xi\right)-D_{\xi} f_{h}\left(x_{2}, \xi\right)\right| \leq c\left|x_{1}-x_{2}\right|^{\alpha}\left(1+\frac{1}{h^{2}}+|\xi|^{2}\right)^{\frac{p-1}{2}} & \text { with } \alpha \in(0,1) ;
\end{array}
$$

$$
f_{h} \rightarrow f \text { uniformly on compact subsets of }
$$

$$
B_{R} \times \mathbb{R}^{n N}
$$

where the number $\nu$ is the same appearing in $(H 1)$ so it is independent of $h$.

Let us observe that since every $f_{h}(x, \cdot) \in C^{2}\left(\mathbb{R}^{n N}\right)$ condition $(i i)$ turns out to be equivalent to $(H 2)$ thanks to Lemma 2.1.

Now we can complete the proof of Theorems 1.1 and 1.2 at once.

Proof of Theorems 1.1 and 1.2. Let us consider, for every $h$, the solution $u_{h}$ of the Dirichlet problem

$$
\min \left\{\int_{B_{R}} f_{h}(x, D v) \mathrm{d} x: v \in u+W_{0}^{1, p}\left(B_{R} ; \mathbb{R}^{N}\right)\right\} .
$$


Thanks to Lemmas 1.1 and 1.2 , the sequence $\left\{u_{h}\right\}$ turns out to be locally bounded in $W^{1, \gamma}\left(B_{R} ; \mathbb{R}^{N}\right)$ for every $k$ in $(0, \alpha)$, where

$$
\begin{array}{rr}
\gamma=\frac{n p}{n-k q} & \text { if } p \geq 2 \\
\gamma=\frac{n p}{n-2 k} & \text { if } 1<p<2 .
\end{array}
$$

Therefore, up to a subsequence, $\left\{u_{h}\right\}$ converges weakly to some $u_{\infty}$ in $W_{\text {loc }}^{1, \gamma}\left(B_{R} ; \mathbb{R}^{N}\right)$; let us prove that $u_{\infty}$ verifies the estimates (1.6) and (1.9). From (i) we have

$$
\begin{aligned}
\left\|D u_{\infty}\right\|_{L^{\gamma}\left(B_{\rho}\right)} & \leq \liminf _{h}\left\|D u_{h}\right\|_{L^{\gamma}\left(B_{\rho}\right)} \leq c \liminf _{h}\left(\int_{B_{R}}\left(1+\left|D u_{h}\right|^{2}\right)^{\frac{p}{2}} \mathrm{~d} x\right)^{\frac{1}{2}} \leq c \liminf _{h}\left(\int_{B_{R}} f_{h}\left(x, D u_{h}\right) \mathrm{d} x\right)^{\frac{1}{2}} \\
& \leq c \liminf _{h}\left(\int_{B_{R}} f_{h}(x, D u) \mathrm{d} x\right)^{\frac{1}{2}} \leq c\left(\int_{B_{R}}\left(1+|D u|^{2}\right)^{\frac{p}{2}} \mathrm{~d} x\right)^{\frac{1}{2}}
\end{aligned}
$$

where we also used the minimality of $u_{h}$.

Now, exploiting the local higher equi-integrability of $\left\{u_{h}\right\}$ which follows from the estimates provided by Lemmas 1.1 and 1.2 , we shall prove that $u_{\infty} \equiv u$. Fixed $M \in \mathbb{N}$ we can consider for every $\rho<R$

$$
\begin{aligned}
\int_{B_{\rho}} f\left(x, D u_{h}\right) \mathrm{d} x= & \int_{B_{\rho} \cap\left\{\left|D u_{h}\right| \leq M\right\}} f\left(x, D u_{h}\right) \mathrm{d} x+\int_{B_{\rho} \cap\left\{\left|D u_{h}\right|>M\right\}} f\left(x, D u_{h}\right) \mathrm{d} x \\
\leq & \int_{B_{\rho} \cap\left\{\left|D u_{h}\right| \leq M\right\}}\left[f\left(x, D u_{h}\right)-f_{h}\left(x, D u_{h}\right)\right] \mathrm{d} x+\int_{B_{\rho}} f_{h}\left(x, D u_{h}\right) \mathrm{d} x \\
& +\int_{B_{\rho} \cap\left\{\left|D u_{h}\right|>M\right\}} f\left(x, D u_{h}\right) \mathrm{d} x .
\end{aligned}
$$

Remembering that $f_{h}$ converges uniformly to $f$ on compact subset (see $(i i i)$ ) we have

$$
\lim _{h} \int_{B_{\rho} \cap\left\{\left|D u_{h}\right| \leq M\right\}}\left[f\left(x, D u_{h}\right)-f_{h}\left(x, D u_{h}\right)\right] \mathrm{d} x=0,
$$

therefore

$$
\liminf _{h} \int_{B_{\rho}} f\left(x, D u_{h}\right) \mathrm{d} x \leq \underset{h}{\limsup } \int_{B_{\rho}} f_{h}\left(x, D u_{h}\right) \mathrm{d} x+\limsup _{h} \int_{B_{\rho} \cap\left\{\left|D u_{h}\right|>M\right\}} f\left(x, D u_{h}\right) \mathrm{d} x .
$$

Moreover, since

$$
\limsup _{h} \int_{B_{\rho} \cap\{|D u| \leq M\}}\left[f_{h}(x, D u)-f(x, D u)\right] \mathrm{d} x=0,
$$

by the minimality of $u_{h}$ we can control the right hand side of (4.1) by

$$
\int_{B_{\rho}} f(x, D u) \mathrm{d} x+\limsup _{h} \int_{B_{\rho} \cap\{|D u|>M\}} f_{h}(x, D u) \mathrm{d} x+\limsup _{h} \int_{B_{\rho} \cap\left\{\left|D u_{h}\right|>M\right\}} f\left(x, D u_{h}\right) \mathrm{d} x .
$$


Using the growth conditions $(i)$ on $f$ and $f_{h}$ we have

$$
\begin{aligned}
\liminf _{h} \int_{B_{\rho}} f\left(x, D u_{h}\right) \mathrm{d} x \leq & \int_{B_{\rho}} f(x, D u) \mathrm{d} x+c L \int_{B_{\rho} \cap\{|D u|>M\}}\left(1+|D u|^{2}\right)^{\frac{p}{2}} \mathrm{~d} x \\
& +c L \limsup _{h} \int_{B_{\rho} \cap\left\{\left|D u_{h}\right|>M\right\}}\left(1+\left|D u_{h}\right|^{2}\right)^{\frac{p}{2}} \mathrm{~d} x,
\end{aligned}
$$

where $L>0$ is the same growth constant appearing in the assumption (H1). Applying Hölder's inequality we can estimate the last integral in (4.2) with

$$
\underset{h}{\limsup c L}\left[\left(\int_{B_{\rho}}\left(1+\left|D u_{h}\right|^{2}\right)^{\frac{\gamma p}{2}} \mathrm{~d} x\right)^{\frac{1}{\gamma}} \cdot\left|\left\{\left|D u_{h}\right|>M\right\} \cap B_{\rho}\right|^{1-\frac{1}{\gamma}}\right]
$$

where the first factor is finite and independent of $h$. So we get the estimate

$$
\begin{aligned}
\liminf _{h} \int_{B_{\rho}} f\left(x, D u_{h}\right) \mathrm{d} x \leq & \int_{B_{\rho}} f(x, D u) \mathrm{d} x+c L\left(\limsup _{h}\left|\left\{\left|D u_{h}\right|>M\right\} \cap B_{\rho}\right|\right)^{1-\frac{1}{\gamma}} \\
& +c L \int_{B_{\rho} \cap\{|D u|>M\}}\left(1+|D u|^{2}\right)^{\frac{p}{2}} \mathrm{~d} x .
\end{aligned}
$$

Note that

$$
\limsup _{M \rightarrow+\infty} \int_{B_{\rho} \cap\{|D u|>M\}}\left(1+|D u|^{2}\right)^{\frac{p}{2}} \mathrm{~d} x=0 \quad \text { and } \quad\left|\left\{\left|D u_{h}\right|>M\right\} \cap B_{\rho}\right| M^{\gamma} \leq \int_{B_{\rho}}\left|D u_{h}\right|^{\gamma} \mathrm{d} x \leq C
$$

where the constant $C$ does not depend on $h$. Therefore

$$
\left|\left\{\left|D u_{h}\right|>M\right\} \cap B_{\rho}\right| \leq \frac{C}{M^{\gamma}}
$$

for every $h$ and

when $M \rightarrow+\infty$. Then we have

$$
\underset{h}{\limsup }\left|\left\{\left|D u_{h}\right|>M\right\} \cap B_{\rho}\right|=0
$$

$$
\int_{B_{\rho}} f\left(x, D u_{\infty}\right) \mathrm{d} x \leq \liminf _{h} \int_{B_{\rho}} f\left(x, D u_{h}\right) \mathrm{d} x \leq \int_{B_{\rho}} f(x, D u) \mathrm{d} x .
$$

Passing to the limit as $\rho \uparrow R$ we can conclude

$$
\int_{B_{R}} f\left(x, D u_{\infty}\right) \mathrm{d} x \leq \int_{B_{R}} f(x, D u) \mathrm{d} x .
$$

Since $u$ is a local minimizer of the functional $\mathcal{F}$ and $u_{\infty} \equiv u$ on the boundary of $B_{R}$, the strict convexity of $f$ implies $u_{\infty} \equiv u$.

Now we can apply Theorems 1.1 and 1.2 to get a bound on the Hausdorff dimension of the singular set $\Sigma$ of minimizers of functional $\mathcal{F}$.

We recall that a local minimizer of $\mathcal{F}$ is partially $C^{1, \mu}$-regular if there exists an open subset of full measure $\Omega_{0} \subseteq \Omega$ such that $D u$ is Hölder continuous in $\Omega_{0}$. 
It is well known that, in general, in the vectorial case local minimizers are not everywhere regular. For counterexamples to any type of regularity in the interior of $\Omega$ (even the $L^{\infty}$ one) of minimizers of regular variational integrals in the vectorial case and solutions to nonlinear elliptic systems see for instance $[8,18,24,26,27]$.

In this situation one can try to prove that the set $\Omega \backslash \Omega_{0}$ is "reasonably small" in the sense that it is not only negligible but it has also a low Hausdorff dimension. Since the singular set of a local minimizer $u$ is contained in the set of non-Lebesgue points of $D u$, the conclusion is achieved by proving an estimate on the size of nonLebesgue point of functions belonging to a fractional order Sobolev space. For a more detailed discussion on partial regularity and singular set see [22].

In our case we have the following result.

Corollary 4.1. If $f$ is a $C^{2}$ function satisfying the assumptions $(H 1),(H 2)$, (H3) and the function $u \in$ $W^{1, p}\left(\Omega ; \mathbb{R}^{N}\right)$ is a local minimizer of $\mathcal{F}$ in $\Omega$, then for the Hausdorff dimension of the singular set $\Sigma$ of the function $u$ the following estimates hold

$$
\begin{array}{rr}
\operatorname{dim}_{\mathcal{H}}(\Sigma) \leq n-\alpha q & \text { if } p \geq 2 ; \\
\operatorname{dim}_{\mathcal{H}}(\Sigma) \leq n-\beta p & \text { if } 1<p<2 ;
\end{array}
$$

where $q=\frac{p}{p-1}$ and $\beta:=\min \left\{\alpha, \frac{1}{2}\right\}$.

Proof. If $u \in W^{1, p}, p>1$, is a local minimizer of the functional $\mathcal{F}$ as a consequence of the Theorems 1.1 and 1.2 we have in particular that

$$
D u \in W^{\frac{k}{p-1}, p} \quad \text { if } p \geq 2 \text {; }
$$

for every $k \in(0, \alpha)$ and

$$
D u \in W^{k, p} \quad \text { if } 1<p<2 ;
$$

for every $k \in(0, \beta)$ where $\beta:=\min \left\{\alpha, \frac{1}{2}\right\}$. Therefore applying Lemma 2.6 we immediately conclude the proof.

\section{REFERENCES}

[1] E. Acerbi and N. Fusco, A regularity theorem for minimizers of quasiconvex integrals. Arch. Ration. Mech. Anal. 99 (1987) $261-281$.

[2] E. Acerbi and N. Fusco, Regularity of minimizers of non-quadratic functionals: the case $1<p<2$. J. Math. Anal. Appl. 140 (1989) 115-135.

[3] R.A. Adams, Sobolev Spaces. Academic Press, New York (1975).

[4] M. Carozza and A. Passarelli di Napoli, A regularity theorem for minimizers of quasiconvex integrals: the case $1<p<2^{\star}$. Proc. R. Math. Soc. Edinb. A 126 (1996) 1181-1199.

[5] M. Carozza and A. Passarelli di Napoli, Model problems from nonlinear elasticity: partial regularity results. ESAIM: COCV 13 (2007) 120-134.

[6] M. Carozza, N. Fusco and R. Mingione, Partial regularity of minimizers of quasiconvex integrals with subquadratic growth. Annali di matematica pura e applicata (IV) CLXXV (1998) 141-164.

[7] G. Cupini, N. Fusco and R. Petti, Hölder continuity of local minimizers. J. Math. Anal. Appl. 235 (1999) 578-597.

[8] E. De Giorgi, Un esempio di estremali discontinue per un problema variazionale di tipo ellittico. Boll. Un. Mat. It. 1 (1968) $135-137$.

[9] L. Esposito, F. Leonetti and G. Mingione, Higher integrability for minimizers of integral functionals with $(p, q)$ growth. J. Differ. Equ. 157 (1999) 414-438.

[10] L. Esposito, F. Leonetti and G. Mingione, Regularity results for minimizers of irregular integrals with $(p, q)$ growth. Forum Math. 14 (2002) 245-272.

[11] L. Esposito, F. Leonetti and G. Mingione, Sharp regularity for functionals with ( $p, q)$ growth. J. Differ. Equ. 204 (2004) 5-55.

[12] L.C. Evans, Quasiconvexity and partial regularity in the Calculus of Variations. Arch. Ration. Mech. Anal. 95 (1984) $227-252$.

[13] L.C. Evans and R.F. Gariepy, Blow-up, compactness and partial regularity in the Calculus of Variations. Indiana Univ. Math. J. 36 (1987) 361-371.

[14] I. Fonseca and N. Fusco, Regularity results for anisotropic image segmentation models. Ann. Scuola Norm. Sup. Pisa Cl. Sci. (4) 3 (1997) 463-499. 
[15] I. Fonseca, N. Fusco and P. Marcellini, An existence result for a nonconvex variational problem via regularity. ESAIM: $C O C V$ 7 (2002) 69-95.

[16] M. Giaquinta and G. Modica, Remarks on the regularity of minimizers of certain degenerate functionals. Manuscripta Math. 47 (1986) 55-99.

[17] E. Giusti, Direct methods in the calculus of variations. World Scientific, River Edge, USA (2003).

[18] O. John, J. Malý and J. Stará, Nowhere continuous solutions to elliptic systems. Comm. Math. Univ. Carolin. 30 (1989) $33-43$.

[19] J. Kristensen and G. Mingione, Non-differentiable functionals and singular sets of minima. C. R. Acad. Sci. Paris Ser. I Math. 340 (2005) 93-98.

[20] J. Kristensen and G. Mingione, The singular set of minima of integral functionals. Arch. Ration. Mech. Anal. 180 (2006) $331-398$.

[21] G. Mingione, The singular set of solutions to non differentiable elliptic systems. Arch. Ration. Mech. Anal. 166 (2003) $287-301$.

[22] G. Mingione, Bounds for the singular set of solutions to non linear elliptic system. Calc. Var. 18 (2003) 373-400.

[23] G. Mingione, Regularity of minima: an invitation to the dark side of calculus of variations. Appl. Math. 51 (2006) 355-426.

[24] J. Nečas, Example of an irregular solution to a nonlinear elliptic system with analytic coefficients and conditions for regularity, in Theory of nonlinear operators, Proc. Fourth Internat. Summer School, Acad. Sci., Berlin (1975) $197-206$.

[25] A. Passarelli di Napoli, A regularity result for a class of polyconvex functionals. Ric. di Matem. XLVIII (1994) 379-393.

[26] V. Šverák and X. Yan, A singular minimizer of a smooth strongly convex functional in three dimensions. Calc. Var. 10 (2000) 213-221.

[27] V. Šverák and X. Yan, Non Lipschitz minimizers of smooth strongly convex variational integrals. Proc. Nat. Acad. Sc. USA 99 (2002) 15269-15276. 